\title{
Nonlinear Periodic Oscillation of a Cylindrical Microvoid Centered at an Isotropic Incompressible Ogden Cylinder
}

\author{
Wenzheng Zhang, ${ }^{1}$ Xuegang Yuan, ${ }^{1,2}$ and Hongwu Zhang1 \\ ${ }^{1}$ State Key Laboratory of Structural Analysis for Industrial Equipment, \\ Department of Engineering Mechanics, Faculty of Vehicle Engineering and Mechanics, \\ Dalian University of Technology, Dalian 116024, China \\ ${ }^{2}$ School of Science, Dalian Nationalities University, Dalian 116600, China \\ Correspondence should be addressed to Xuegang Yuan, yxg1971@163.com
}

Received 31 August 2012; Accepted 5 December 2012

Academic Editor: Kale Oyedeji

Copyright (C 2012 Wenzheng Zhang et al. This is an open access article distributed under the Creative Commons Attribution License, which permits unrestricted use, distribution, and reproduction in any medium, provided the original work is properly cited.

\begin{abstract}
We study the dynamic mathematical model for an infinitely long cylinder composed of an isotropic incompressible Ogden material with a microvoid at its center, where the outer surface of the cylinder is subjected to a uniform radial tensile load. Using the incompressibility condition and the boundary conditions, we obtain a second-order nonlinear ordinary differential equation that describes the motion of the microvoid with time. Qualitatively, we find that this equation has two types of solutions. One is a classical nonlinear periodic solution which describes that the motion of the microvoid is a nonlinear periodic oscillation; the other is a blow-up solution. Significantly, for the isotropic incompressible Ogden material, there exist some special values of material parameters, the phase diagrams of the motion equation have homoclinic orbits, which means that the amplitude of a nonlinear periodic oscillation increases discontinuously with the increasing load.
\end{abstract}

\section{Introduction}

Cylindrical structures are very common used in social productions and human lives. The researches on the dynamic oscillation problems of such structures composed of hyperelastic materials are of important significance. As is well known, such problems can be formulated as initial (boundary) value problems of nonlinear evolution equation(s). Knowles [1] firstly studied the free radial oscillation of an incompressible cylindrical tube composed of an isotropic Mooney-Rivlin material; in the limiting case of a thin walled cylindrical tube, the equation reduces to the Ermakov-Pinney equation. Then, Shahinpoor and Nowinski 
[2] and Rogers and Baker [3] used the nonlinear superposition principle for the ErmakovPinney equation to derive solutions. The works appeared in this area have been reviewed by Rogers and Ames [4]. In 2007, Mason and Maluleke [5] introduced the Lie point symmetry into this area and investigated the nonlinear radial oscillations of a transversely isotropic incompressible cylindrical tube subjected to time dependent net applied surface pressures; moreover, they proved that for radial and tangential transversely isotropic tubes the differential equations may be reduced to the Abel equations of the second kind. In addition, with the development of the mathematical theory, Yuan et al. [6] investigated the dynamic inflation problems for infinitely long cylindrical tubes composed of a class of transversely isotropic incompressible Ogden materials from the equation itself and discussed the influences of material parameters, structure parameter and applied pressures on the dynamic behaviors of the tubes in detail. Ren [7] studied the dynamical responses, such as motion and destruction of hyperelastic cylindrical shells subjected to dynamic loads on the inner surface. Other references on the dynamic responses for hyperelastic cylindrical structures may be found in Dai and Kong [8], Yuan et al. [9], and so on.

The purpose of this paper is to investigate the nonlinear periodic oscillation of a cylindrical microvoid centered at an infinitely long cylinder, where the cylinder is composed of an isotropic incompressible Ogden material [10] and its outer surface is subjected to a uniform radial tensile load. In Section 2, the basic governing equations, the boundary conditions and the initial conditions are presented. In Section 3, a second order nonlinear ordinary differential equation describing the motion of the microvoid is obtained. Then, in Section 4, some nonlinear dynamic analyses of the equation are performed in detail. Meanwhile, some numerical examples are given.

\section{Mathematical Model}

The mathematical model examined in this paper is listed as follows.

\section{(a) Basic Governing Equations}

In the absence of body force, the equilibrium differential equation, the incompressibility condition and the strain-energy function associated with the known Ogden material are given by

$$
\begin{gathered}
\frac{\partial \sigma_{r}(r, t)}{\partial r}+\frac{1}{r}\left(\sigma_{r}(r, t)-\sigma_{\theta}(r, t)\right)=\rho_{0} \frac{\partial^{2} r(R, t)}{\partial t^{2}}, \\
\lambda_{r} \lambda_{\theta}=1 \\
W=\frac{\mu_{1}}{\alpha_{1}}\left[\lambda_{r}^{\alpha_{1}}+\lambda_{\theta}^{\alpha_{1}}-2\right]+\frac{\mu_{2}}{\alpha_{2}}\left[\lambda_{r}^{\alpha_{2}}+\lambda_{\theta}^{\alpha_{2}}-2\right] .
\end{gathered}
$$

\section{(b) Boundary Conditions and Initial Conditions}

The boundary conditions are given by

$$
\sigma_{r}(r(A), t)=0, \quad \sigma_{r}(r(B), t)=p_{0}\left[\frac{B}{r(B)}\right] .
$$


The initial conditions are as follows:

$$
r(R, 0)=R, \quad \dot{r}(R, 0)=0 .
$$

In (2.1) (2.5), $\sigma_{i}(r, t)=\lambda_{i}\left(\partial W / \partial \lambda_{i}\right)-p(r, t), i=r, \theta$ are the principal Cauchy stresses, $p(r, t)$ is the hydrostatic pressure related to the incompressibility condition, $\lambda_{r}=\partial r / \partial R, \lambda_{\theta}=$ $r / R$ are the radial and the circumference stretches, respectively, $r=r(R, t), 0<A \leq R \leq B$ is the radial deformation function with time to be determined, and $A$ and $B$ are the radius of the microvoid and the outer radius of the cylinder in the undeformed configuration, respectively. $\rho_{0}$ is the constant mass density of the material. $\mu_{i}, \alpha_{i}(i=1,2)$ are material parameters. The boundary conditions in (2.4) mean that the surface of the microvoid is traction free and the outer surface of the cylinder is subjected to a uniform radial tensile load, denoted by $p_{0}$. The initial conditions in (2.5) mean that the cylinder is in an undeformed state at time $t=0$.

\section{Solutions}

From the incompressibility condition (2.2) we find that

$$
r(R, t)=\left(R^{2}-A^{2}+r_{1}^{2}(t)\right)^{1 / 2},
$$

where $r_{1}(t)$ is an undetermined radial motion function of the radius of the microvoid. From (3.1), it is easy to know that the radial motion of the cylinder can be completely described by $r_{1}(t)$.

From (3.1), it is not difficult to show that

$$
\frac{\partial^{2} r(R, t)}{\partial t^{2}}=\frac{\partial}{\partial r}\left(r_{1} \ddot{r}_{1} \ln r+\left(\dot{r}_{1}\right)^{2} \ln r+\frac{1}{2}\left(\dot{r}_{1}\right)^{2}\left(\frac{r_{1}}{r}\right)^{2}\right) .
$$

Substituting (3.2) into (2.1), integrating it with respect to $r$ from $r(A, t)$ to $r(B, t)$ and using the traction boundary conditions in (2.4), we obtain

$$
\begin{gathered}
\frac{1}{2} \rho_{0} r_{1} \ddot{r}_{1} \ln \left(\frac{B^{2}-A^{2}+r_{1}^{2}}{r_{1}^{2}}\right)+\frac{1}{2} \rho_{0} r_{1}^{2} \ln \left(\frac{B^{2}-A^{2}+r_{1}^{2}}{r_{1}^{2}}\right) \\
+\frac{1}{2} \rho_{0} \dot{r}_{1}^{2}\left(\frac{A^{2}-B^{2}}{B^{2}-A^{2}+r_{1}^{2}}\right)-p_{0}\left(\frac{B}{\left(B^{2}-A^{2}+r_{1}^{2}\right)^{1 / 2}}\right) \\
-\int_{r(A, t)}^{r(B, t)}\left[\lambda_{r} \frac{\partial W}{\partial \lambda_{r}}-\lambda_{\theta} \frac{\partial W}{\partial \lambda_{\theta}}\right] \frac{d r}{r}=0,
\end{gathered}
$$

where $r(A, t)=\left(A^{2}-A^{2}+r_{1}^{2}(t)\right)^{1 / 2}=r_{1}(t), r(B, t)=\left(B^{2}-A^{2}+r_{1}^{2}(t)\right)^{1 / 2}$.

Obviously, (3.3) is a second order nonlinear ordinary differential equation with respect to $r_{1}(t)$. Next we study the qualitative properties of solutions of (3.3). 
For convenience, we introduce the following dimensionless notations:

$$
\begin{gathered}
k=\left(\frac{R}{r}\right)^{-1}=\left(1-\frac{r_{1}^{2}-A^{2}}{r^{2}}\right)^{-1 / 2}, \quad x(t)=\frac{r_{1}(t)}{B}, \\
\dot{x}(t)=\frac{\dot{r}_{1}(t)}{B}, \quad \delta=\frac{\left(B^{2}-A^{2}\right)^{1 / 2}}{B} .
\end{gathered}
$$

Thus, the initial conditions in (2.5) reduce to

$$
x(0)=\left(1-\delta^{2}\right)^{1 / 2}, \quad \dot{x}(0)=0,
$$

and (3.3) may be rewritten as

$$
\begin{gathered}
\frac{1}{2} \rho_{0} B^{2} x \ddot{x} \ln \left(\frac{\delta^{2}+x^{2}}{x^{2}}\right)+\frac{1}{2} \rho_{0} B^{2} \dot{x}^{2} \ln \left(\frac{\delta^{2}+x^{2}}{x^{2}}\right)-\frac{1}{2} \rho_{0} B^{2} \dot{x}^{2}\left(\frac{\delta^{2}}{\delta^{2}+x^{2}}\right) \\
-p_{0}\left(\frac{1}{\delta^{2}+x^{2}}\right)^{1 / 2}+F(x, \delta)=0,
\end{gathered}
$$

where

$$
F\left(x, \delta, \alpha_{1}, \alpha_{2}, \mu_{1}, \mu_{2}\right)=\int_{x /\left(1-\delta^{2}\right)^{1 / 2}}^{\left(\delta^{2}+x^{2}\right)^{1 / 2}} \frac{\mu_{1} k^{-\alpha_{1}}+\mu_{2} k^{-\alpha_{2}}-\mu_{1} k^{\alpha_{1}}-\mu_{2} k^{\alpha_{2}}}{k\left(k^{2}-1\right)} d k
$$

\section{Nonlinear Dynamic Analyses}

Multiplying (3.6) by $x \dot{x}$, we obtain the following first integral:

$$
U(x, \delta) \dot{x}^{2}+V\left(x, p_{0}, \delta\right)=0,
$$

where

$$
\begin{gathered}
U(x, \delta)=\frac{1}{4} \rho_{0} B^{2} x^{2} \ln \left(\frac{\delta^{2}+x^{2}}{x^{2}}\right) \\
V\left(x, p_{0}, \delta\right)=\int_{\left(1-\delta^{2}\right)^{1 / 2}}^{x}\left(F(s, \delta)-p_{0}\left(\frac{1}{\delta^{2}+s^{2}}\right)^{1 / 2}\right) s d s .
\end{gathered}
$$




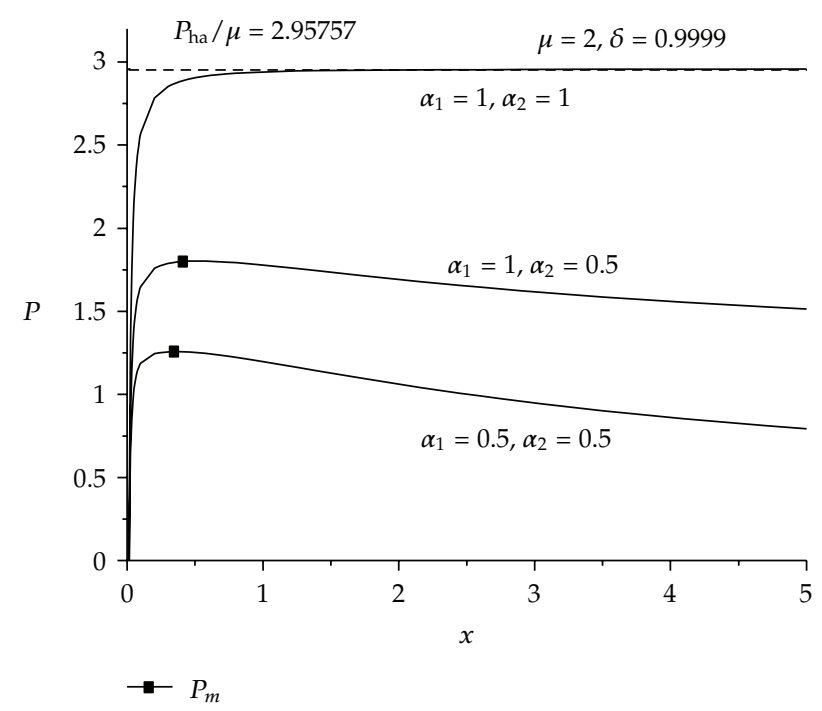

Figure 1: Curves of $P \sim x$ for $0<\alpha_{1}, \alpha_{2} \leq 1$.

Attentively, $U(x, \delta)>0$ is valid for any $x>\left(1-\delta^{2}\right)^{1 / 2}$, which means that (4.1) has real solutions only when $V\left(x, p_{0}, \delta\right)<0$. However, $V\left(x, p_{0}, \delta\right)<0$ is equivalent to $V\left(x, p_{0}, \delta\right)_{\min }<$ 0 . The stationary point of $V\left(x, p_{0}, \delta\right)$ can be obtained from $V_{x}\left(x, p_{0}, \delta\right)=0$, this leads to

$$
\begin{aligned}
P & =\left(\delta^{2}+x^{2}\right)^{1 / 2} \int_{x /\left(1-\delta^{2}\right)^{1 / 2}}^{\left(\delta^{2}+x^{2}\right)^{1 / 2}} \frac{k^{-\alpha_{1}}+\mu k^{-\alpha_{2}}-k^{\alpha_{1}}-\mu k^{\alpha_{2}}}{k\left(k^{2}-1\right)} d k \\
& =G\left(x, \delta, \alpha_{1}, \alpha_{2}, \mu\right),
\end{aligned}
$$

where $P=p_{0} / \mu_{1}, \mu=\mu_{2} / \mu_{1}$.

Interestingly, the equilibrium points of (3.6) can be determined by the roots of (4.3).

\subsection{Influences of Parameters on the Solution of (3.6)}

\subsubsection{Influences of Material Parameters}

The following conclusions can be obtained from (4.3).

(i) For the given values of $\mu$ and $\delta$, if $0<\alpha_{1}, \alpha_{2}<1$, there exists a maximum point, written as $\left(x_{m}, P_{m}\right) . P$ increases monotonically as $0<x<x_{m}$ and decreases monotonically as $x>x_{m}$.

(ii) For the case that $\alpha_{1}=\alpha_{2}=1$, we have $\lim _{x \rightarrow+\infty} G(x, \delta, 1,1, \mu)=(1+\mu)\left(1-\left(1-\delta^{2}\right)^{1 / 2}\right)$, which means (4.3) has a horizontal asymptote, written as $P_{\text {ha }}=(1+\mu)(1-$ $\left.\left(1-\delta^{2}\right)^{1 / 2}\right)$. For the given values of $\mu$ and $\delta$, curves of $P$ versus $x$ are shown in Figure 1 for $0<\alpha_{1}, \alpha_{2} \leq 1$. 


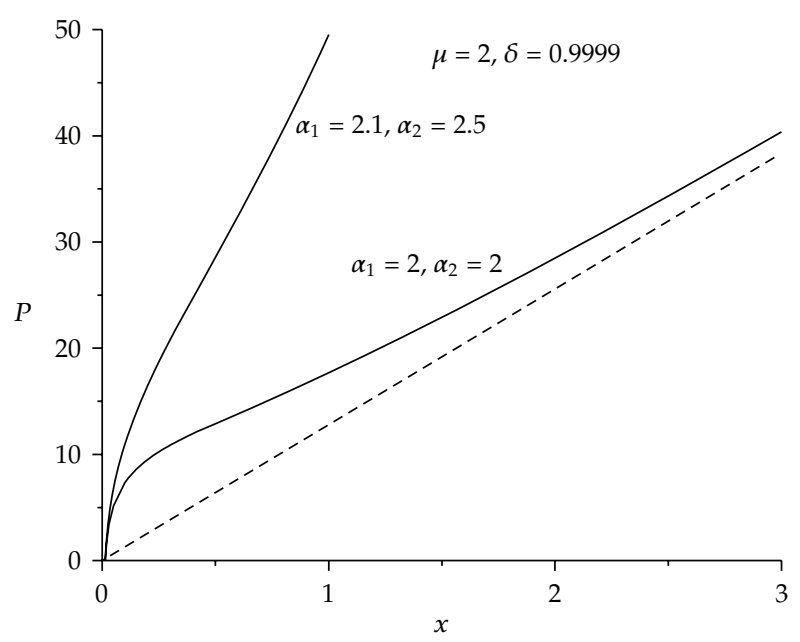

Figure 2: Curves of $P \sim x$ for $\alpha_{1}, \alpha_{2}>1$.

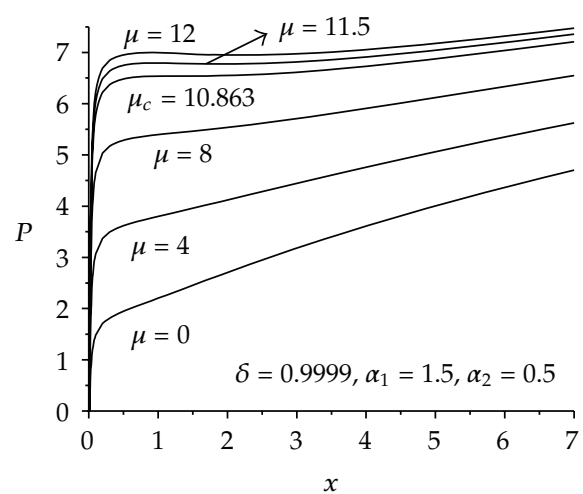

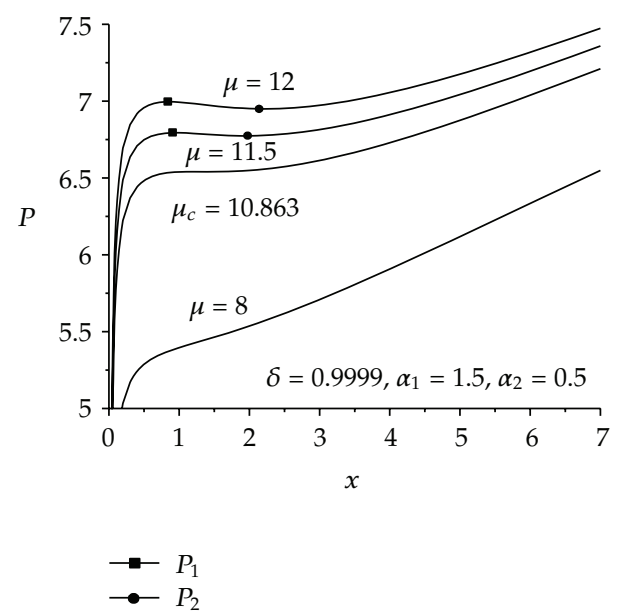

(b)

(a)

Figure 3: Curves of $P \sim x$ for $\alpha_{1}>1,0<\alpha_{2}<1$.

(iii) If $\alpha_{1}, \alpha_{2}>1$, we have $\lim _{x \rightarrow+\infty} G\left(x, \delta, \alpha_{1}, \alpha_{2}, \mu\right)=+\infty$. $P$ increases strictly with the increasing $x$. Particularly, if $\alpha_{1}=\alpha_{2}=2$, it is easy to prove that there is another asymptote, written as $P_{a}(x)=-((1+\mu) / 2) \ln \left(1-\delta^{2}\right) x$. For the given values of $\mu$ and $\delta$, Figure 2 shows the relationships of $P$ versus $x$ for $\alpha_{1}, \alpha_{2}>1$.

(iv) For the case that $\alpha_{1}>1,0<\alpha_{2}<1$ or $0<\alpha_{1}<1, \alpha_{2}>1$ (here, we only discuss the case that $\alpha_{1}>1,0<\alpha_{2}<1$ ), it can be proved that there exists a critical value of $\mu$, written as $\mu_{c}$, such that $P$ increases monotonically if $0 \leq \mu<\mu_{c}$ and has a local maximum and a local minimum if $\mu>\mu_{c}$, written as $P_{1}$ and $P_{2}$, respectively. Curves of $P$ versus $x$ are given in Figure 3 for $\alpha_{1}>1,0<\alpha_{2}<1$ and for the given values of $\delta$. 


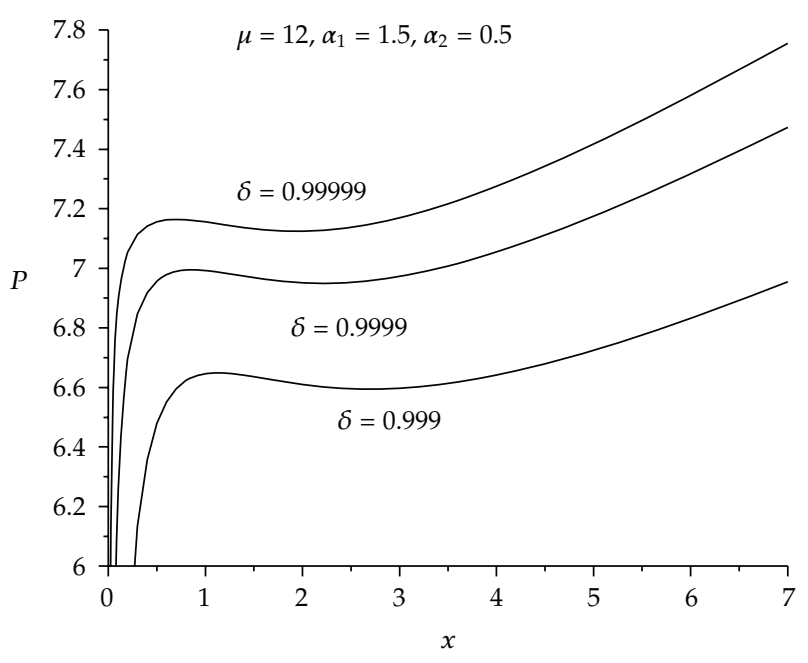

Figure 4: Curves of $P \sim x$ for different values of $\delta$.

\subsubsection{Influence of Structure Parameter}

Once the values of $\alpha_{1}, \alpha_{2}$, and $\mu$ are given, the influence of $\delta$ on the relationships of $P$ versus $x$ is shown in Figure 4.

\subsection{Number of Equilibrium Points}

(1) For the case that $0<\alpha_{1}, \alpha_{2}<1$, it can be seen from Figure 1 that there are two different roots of (4.3) as $0<P<P_{m}$, written as $x_{1}$ and $x_{2}\left(x_{1}<x_{2}\right)$. It means that (3.6) has two equilibrium points $\left(x_{1}, 0\right)$ and $\left(x_{2}, 0\right)$; moreover, $\left(x_{1}, 0\right)$ is a center and $\left(x_{2}, 0\right)$ is a saddle point.

(2) If $\alpha_{1}=\alpha_{2}=1$, (3.6) has a unique equilibrium point as $0 \leq P<P_{\text {ha, }}$, written as $\left(x_{3}, 0\right)$; moreover, $\left(x_{3}, 0\right)$ is a center. While $P>P_{\text {ha }},(3.6)$ has no equilibrium point.

(3) For the case that $\alpha_{1}, \alpha_{2}>1$, (3.6) has only one equilibrium point as shown in Figure 2, written as $\left(x_{4}, 0\right)$, and it is also a center.

(4) If $\alpha_{1}>1,0<\alpha_{2}<1$, from the above analyses we know that $P$ has a local maximum $P_{1}$ and a local minimum $P_{2}$ as $\mu>\mu_{c}$, where $\mu_{c}$ is a critical value of $\mu$. Equation (3.6) has a unique equilibrium point as $P>P_{1}$ or $P<P_{2}$, written as $\left(x_{5}, 0\right)$, and it is a center. Equation (3.6) has exactly three equilibrium points as $P_{1}>P>P_{2}$, written as $\left(x_{6}, 0\right),\left(x_{7}, 0\right)$, and $\left(x_{8}, 0\right)$, respectively, where $x_{6}<x_{7}<x_{8}$; moreover, $\left(x_{6}, 0\right)$ and $\left(x_{8}, 0\right)$ are centers and $\left(x_{7}, 0\right)$ is a saddle point. For the given values of $\mu, \alpha_{1}, \alpha_{2}$, and $\delta$, the phase diagrams of (4.1) are shown in Figure 5. It is found that the radial oscillation of the microvoid presents a nonlinear periodic oscillation; moreover, the amplitude of the oscillation increases gradually as $P$ increases from 0 to $P_{\mathrm{cr}}$. However, the increase of the amplitude of the nonlinear periodic oscillation is discontinuous as $P$ passes through $P_{\mathrm{cr}}$. Another interesting phenomenon occurs as $P=P_{\mathrm{cr}}$, namely, the phase diagram is a homoclinic orbit at the moment. 


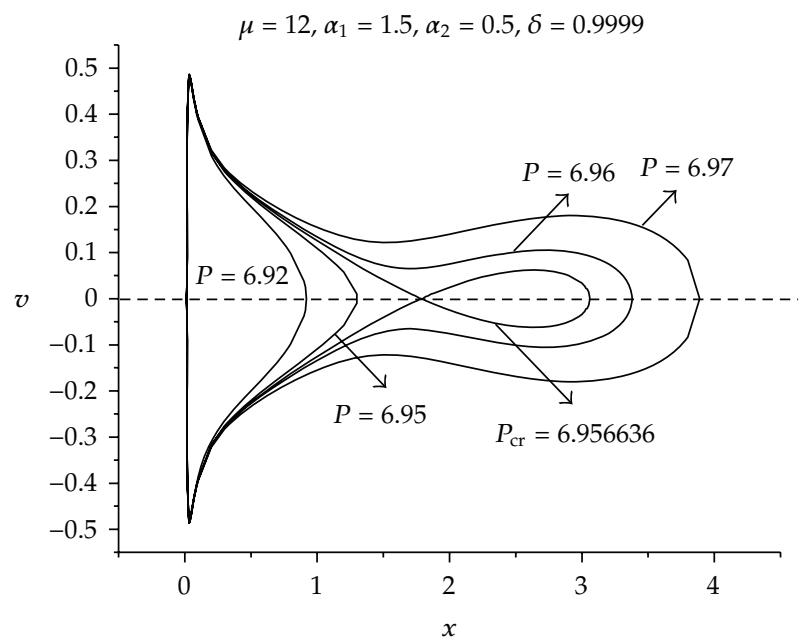

Figure 5: Phase diagrams for different values of $P$ and for $\mu=12, \alpha_{1}=1.5, \alpha_{2}=0.5$, and $\delta=0.9999$.

\section{Conclusions}

In this paper, the nonlinear periodic oscillation of a cylindrical microvoid centered at the isotropic incompressible Ogden cylinder is examined. Some interesting nonlinear dynamic properties of the mathematical model are obtained. The main conclusions are as follows.

(1) If $\alpha_{1}>1,0<\alpha_{2}<1$, it is proved that the motion of the microvoid is the nonlinear periodic oscillation for any given load, but there exist some special values of material parameters, the phase diagrams of the motion equation have homoclinic orbits, which means that the amplitude of the nonlinear periodic oscillation increases discontinuously with the increasing load.

(2) For the case that $\alpha_{1}, \alpha_{2} \geq 1$, the radial oscillation of the microvoid always presents a nonlinear periodic oscillation.

\section{Acknowledgments}

This work was supported by the National Natural Science Foundation of China (nos. 10872045, 11232003); the Program for New Century Excellent Talents in University (no. NCET-09-0096); the Fundamental Research Funds for the Central Universities (no. DC12010112); the Program for Liaoning Excellent Talents in University (no. LR2012044).

\section{References}

[1] J. K. Knowles, "Large amplitude oscillations of a tube of incompressible elastic material," Quarterly of Applied Mathematics, no. 18, pp. 71-77, 1960.

[2] M. Shahinpoor and J. L. Nowinski, "Exact solution to the problem of forced large amplitude radial oscillations of a thin hyperelastic tube," International Journal of Non-Linear Mechanics, vol. 6, no. 2, pp. 193-207, 1971.

[3] C. Rogers and J. A. Baker, "The finite elastodynamics of hyperelastic thin tubes," International Journal of Non-Linear Mechanics, vol. 15, no. 3, pp. 225-233, 1980. 
[4] C. Rogers and W. F. Ames, Nonlinear Boundary Value Problems in Science and Engineering, Academic Press, New York, NY, USA, 1989.

[5] D. P. Mason and G. H. Maluleke, "Nonlinear radial oscillations of a transversely isotropic hyperelastic incompressible tube," Journal of Mathematical Analysis and Applications, vol. 333, no. 1, pp. 365-380, 2007.

[6] X. G. Yuan, R. J. Zhang, and H. W. Zhang, "Controllability conditions of finite oscillations of hyperelastic cylindrical tubes composed of a class of ogden material models," Computers, Materials and Continua, vol. 7, no. 3, pp. 155-156, 2008.

[7] J. S. Ren, "Dynamical response of hyper-elastic cylindrical shells under periodic load," Applied Mathematics and Mechanics-English Edition, vol. 29, no. 10, pp. 1319-1327, 2008.

[8] H. H. Dai and D. X. D. X. Kong, "Global structure stability of impact-induced tensile waves in a rubber-like material," IMA Journal of Applied Mathematics, vol. 71, no. 1, pp. 14-33, 2006.

[9] X. Yuan, W. Zhang, and H. Zhang, "Stability analysis of radial inflation of incompressible composite rubber tubes," Applied Mathematics and Mechanics-English Edition, vol. 32, no. 3, pp. 301-308, 2011.

[10] R. W. Ogden, "Large deformation isotropic elasticity-on the correlation of theory and experiment for incompressible rubberlike solids," Proceedings of the Royal Society of London A, vol. 326, no. 1567, pp. 565-584, 1972. 


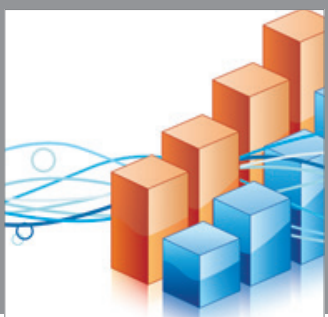

Advances in

Operations Research

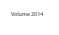

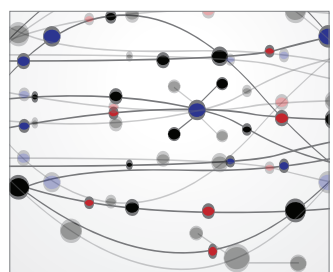

\section{The Scientific} World Journal


International Journal of

Mathematics and

Mathematical

Sciences


Journal of

Applied Mathematics
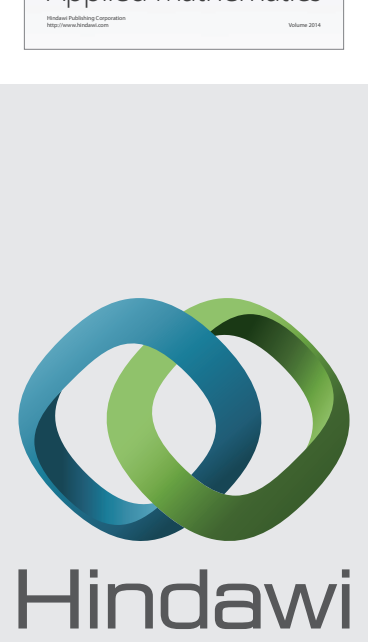

Submit your manuscripts at http://www.hindawi.com
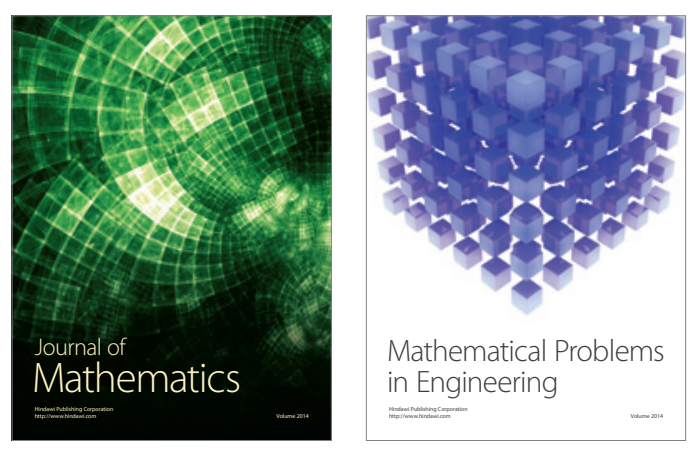

Mathematical Problems in Engineering
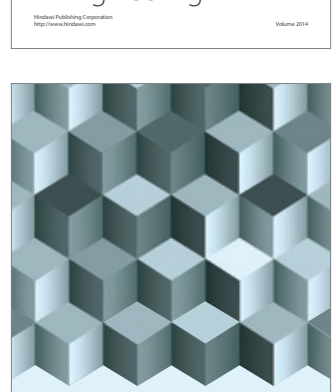

Journal of

Function Spaces
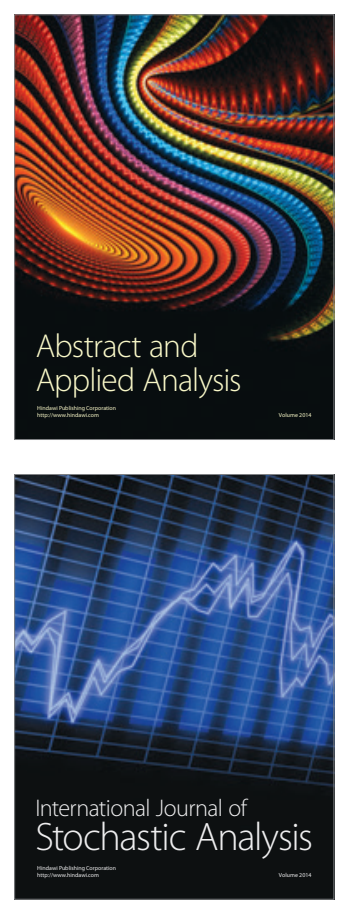

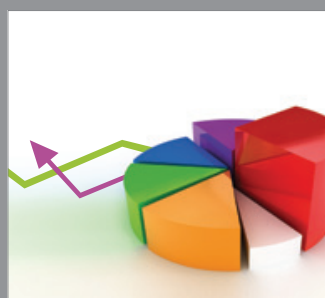

ournal of

Probability and Statistics

Promensencen
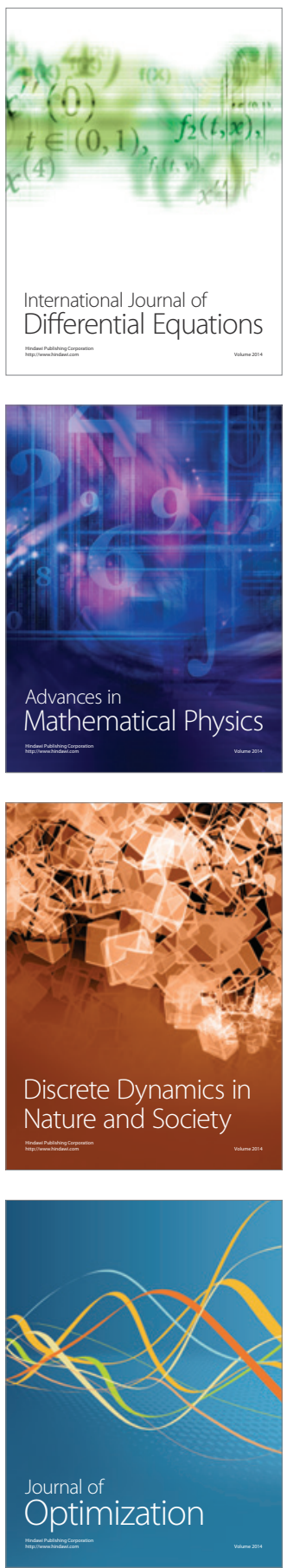